\title{
Queues with Lévy input and hysteretic control
}

\author{
R. Bekker
}

Received: 27 November 2008 / Revised: 25 May 2009 / Published online: 16 June 2009

(C) The Author(s) 2009. This article is published with open access at Springerlink.com

\begin{abstract}
We consider a (doubly) reflected Lévy process where the Lévy exponent is controlled by a hysteretic policy consisting of two stages. In each stage there is typically a different service speed, drift parameter, or arrival rate. We determine the steady-state performance, both for systems with finite and infinite capacity. Thereby, we unify and extend many existing results in the literature, focusing on the special cases of $\mathrm{M} / \mathrm{G} / 1$ queues and Brownian motion.
\end{abstract}

Keywords M/G/1 queue · Storage process · Lévy process · Lévy exponent · Double reflection · Workload distribution · Two-state strategy · Hysteretic control

Mathematics Subject Classification (2000) 60K25 · 60J30

\section{Introduction}

In this paper, we consider a (doubly) reflected spectrally positive Lévy process. The system can be in two stages, where the stage of the system is controlled using a hysteretic policy. If the system is in stage $i, i=1,2$, the Lévy exponent of the driving process $X$ is $\phi_{i}(\cdot)$. The control rule is specified by two switch-over levels $0 \leq m_{2} \leq m_{1}$; the stage of the system changes only at upcrossings of the reflected process ("workload process") of $m_{1}$ while the stage is 1 and at downcrossings of $m_{2}$ while the stage is 2 . For example, let $i=1,2$ be the stage of the system and consider the classical M/G/1 queue with service speed $r_{i}$, arrival rate $\lambda_{i}$, and service time distribution $B_{i}(\cdot)$. The net input process of the queue in stage $i$ is then a compound

R. Bekker $(\bowtie)$

Department of Mathematics, VU University Amsterdam, De Boelelaan 1081a, 1081 HV Amsterdam, The Netherlands

e-mail: rbekker@few.vu.nl 
Poisson process with a negative drift, which is a special case of a spectrally positive Lévy process.

Queueing systems with state-dependent parameters naturally arise as models for congestion phenomena in production and communication systems. In the literature, many of these studies on queueing systems with state-dependent parameters have been devoted to hysteretic control, see e.g. Dshalalow [15] for an extensive survey. Most often, the control parameter consists of an adaptable service speed (i.e. $\lambda_{1}=$ $\lambda_{2}$ and $B_{1}(\cdot)=B_{2}(\cdot)$ ) or an adaptable arrival rate (i.e. $r_{1}=r_{2}$ and $B_{1}(\cdot)=B_{2}(\cdot)$ ). The latter can be equivalently interpreted as admission control. Queueing systems where the service requirement distribution can be adapted based on the state of the system received much less attention, see e.g. [13, 30]. Finally, we refer to [34] for an interesting related paper on hysteretic control for inventory models of the M/G/1type.

From an historical perspective, dams and storage processes also form an important area of systems with state-dependent parameters. Typically, the input process of a large class of dams is a nondecreasing Lévy process and the release rate may be a function of the buffer content, see e.g. [12, 31], or [29, Chap. 3]. Moreover, in the study of dams and storage processes, quite a number of papers appeared using the terminology $P_{\lambda, \tau}^{M}$ policy to describe the release rule of the dam. In fact, the $P_{\lambda, \tau}^{M}$ release policy is the equivalent of hysteretic service-rate control in queueing systems, where $m_{2}, m_{1}$, and $r_{2}$ should be read for the parameters $\lambda, \tau$, and $M$ in the $P_{\lambda, \tau}^{M}$ policy, respectively. In $[16,35]$, the authors considered the optimal control where the input process of the dam is a Wiener process (Brownian motion). For some studies of M/G/1 queues with $P_{\lambda, \tau}^{M}$ policies, we refer to e.g. [5, 26].

The class of spectrally one-sided Lévy processes is much richer than the special cases of Brownian motion and compound Poisson with negative drift (M/G/1 queues). We refer to e.g. [23] and the references therein for an excellent introduction and further examples.

In this paper we consider the steady-state distribution and Laplace-Stieltjes transform (LST), assuming that they exist, of the level of the workload process. The main goal is to present a unified treatment and derive intuitively appealing results for the steady-state workload distribution for the general model with two Lévy exponents, which extends the models in the literature for hysteretic control based on workloads or buffer contents.

Furthermore, we consider both reflected and doubly reflected processes, corresponding to systems with infinite and finite capacity, respectively. Both types of systems are often considered in the literature, but they are commonly treated independently. In the present case of hysteretic control, we derive a proportionality relation between systems with finite and infinite buffer capacity based on martingale arguments. This proportionality is well known for classical queueing models, see e.g. [1, 14]. More recently, this proportionality relation was extended to (doubly) reflected Lévy processes (but without any control), see [28] and Theorem 2.2. An alternative derivation of Theorem 2.2 of independent interest, based on the Kella-Whitt martingale, may be found in Appendix A.

The organization of the paper is as follows. Section 2 presents some preliminary results on reflected Lévy processes. In Sect. 3 we analyze the steady-state distribution 
and LST of the process. In two subsections, we consider the intervals during which the process is in stage 1 and 2, respectively, and present the resulting steady-state distributions and LSTs. The steady-state distributions become even more tractable in various special cases. In Sect. 4, we consider the cases of different drifts in the two stages, the compound Poisson process with negative drift (corresponding to an M/G/1 queue), and Brownian motion. Finally, in Sect. 5, we derive a proportionality relation between the reflected and doubly reflected processes under hysteretic control, corresponding to systems with infinite and finite capacity.

\section{Preliminary results on Lévy processes}

Consider a spectrally positive Lévy process $X=\{X(t), t \geq 0\}$, i.e. a Lévy process with no negative jumps. In this paper, we exclude the cases where $X$ is nondecreasing (a subordinator) or a deterministic (negative) drift. Since $X$ has no negative jumps, the moment generating function of $X(t)$ is finite for all $\alpha \geq 0$ and $t \geq 0$ and is given by

$$
\mathbb{E}\left[\mathrm{e}^{-\alpha X(t)}\right]=\mathrm{e}^{t \phi(\alpha)},
$$

for some function $\phi(\alpha)$, which is called the Lévy exponent. It is well known that $\phi(\alpha)$ is strictly convex on $[0, \infty), \phi(0)=0$, and $\lim _{\alpha \rightarrow \infty} \phi(\alpha)=\infty$, see e.g. [9, 23].

We first introduce the so-called scale function. In fact, this corresponds to the case $q=0$ in the class of $q$-scale functions, which is usually encountered in the literature. Since we are only interested in 'just' the scale function, we restrict the definition to the case $q=0$ here. Let $\Phi(0)$ denote the largest root of $\phi(\alpha)$ on the positive half-axis.

Definition 2.1 The scale function $W:(-\infty, \infty) \rightarrow[0, \infty)$ is the unique function whose restriction to $(0, \infty)$ is continuous and has Laplace transform

$$
\int_{0}^{\infty} \mathrm{e}^{-\alpha x} W(x) \mathrm{d} x=\frac{1}{\phi(\alpha)}, \quad \text { for } \alpha>\Phi(0),
$$

and $W(x)=0$ for $x<0$.

Scale functions play an important role in the study of first-exit times and exit positions. Due to the equivalence between the steady-state distributions of reflected processes and exit problems for their dual processes with absorbing barriers, scale functions can also be used to describe the steady-state distribution of some reflected processes, see also [23]. A typical example of this duality is the two-sided reflected process, which will be discussed below.

Exit problems In the literature, exit problems are often considered in the setting of Lévy processes with only negative jumps, see for instance [10, 24]. The case of Lévy processes with only positive jumps is however directly related. Denote the dual process $\widehat{X}=-X$. Hence, it trivially follows that $\widehat{X}$ is a Lévy process without positive jumps and $\mathbb{E}\left[\mathrm{e}^{\alpha \widehat{X}(t)}\right]=\mathrm{e}^{t \phi(\alpha)}$. 
A specific example where the scale function defined above appears is in the study of two-sided exit problems for the free Lévy process. Define $T_{a}=\inf \{t \geq 0: X(t) \geq$ $a\}$ and $\widehat{T}_{a}=\inf \{t \geq 0:-X(t) \geq a\}$. The law of the Lévy process started at $x \geq 0$ will be denoted by $\mathbb{P}_{x}$. Starting at $x$, the probability that the process $X$ leaves $[0, a]$ at the lower boundary can be obtained by taking $q=0$ in e.g. [24, (5)]:

$$
\mathbb{P}_{x}\left(\widehat{T}_{0}<T_{a}\right)=\frac{W(a-x)}{W(a)} .
$$

In this paper, we are mainly concerned with the exit position of the reflected process as it leaves $[0, a)$. The joint transform of the first-exit time from the interval $[0, a)$ of the reflected process and the exit position of the reflected process can be found in [4, 27]. In [7], this transform is rewritten in the case that only the exit position is required. In Corollary 2.1, we restate the latter result in terms of Lévy processes with only positive jumps.

Throughout, we use the notations $c \vee 0=\max \{c, 0\}$ and $c \wedge 0=\min \{c, 0\}$. The reflected Lévy process is now defined as $Z(t)=X(t)-I(t)$, where $I(t)=$ $\inf _{0 \leq s \leq t}\{X(s) \wedge 0\}$. It follows directly that the process $\widehat{X}$ reflected at its supremum, i.e., $\widehat{Z}(t)=\sup _{0 \leq s \leq t}\{\widehat{X}(s) \vee 0\}-\widehat{X}$, is identical (see also [23]). In particular, from the above identities it follows that an initial position $z$ of the process $Z$ corresponds to an initial maximum $z$ of the process $\widehat{Z}$. Let $\tau_{a}=\inf \{t \geq 0: Z(t) \notin[0, a)\}$ be the firstexit time from $[0, a)$. The transform of its exit position is given in [7, Corollary 2.1]:

Corollary 2.1 For $\alpha \geq 0$, with $X(0)=z \geq 0$,

$$
\begin{aligned}
\mathbb{E}_{z}\left[\mathrm{e}^{-\alpha Z\left(\tau_{a}\right)}\right]= & \mathrm{e}^{-\alpha z}\left(1-\phi(\alpha) \int_{0}^{a-z} \mathrm{e}^{-\alpha y} W(y) \mathrm{d} y\right) \\
& -\frac{W(a-z)}{W^{\prime}(a)}\left(\alpha-\phi(\alpha) \int_{0}^{a} \mathrm{e}^{-\alpha y} \mathrm{~d} W(y)\right),
\end{aligned}
$$

which, for $\alpha>\Phi(0)$, can be reduced to

$$
\mathbb{E}_{z}\left[\mathrm{e}^{-\alpha Z\left(\tau_{a}\right)}\right]=\phi(\alpha)\left(\mathrm{e}^{-\alpha z} \int_{a-z}^{\infty} \mathrm{e}^{-\alpha y} W(y) \mathrm{d} y-\frac{W(a-z)}{W^{\prime}(a)} \int_{a}^{\infty} \mathrm{e}^{-\alpha y} \mathrm{~d} W(y)\right) .
$$

Steady-state results We now turn to the steady-state workload (denoted by $Y$ ) of the reflected Lévy process $Z$. The formula for its LST is well known as the generalized Pollaczek-Khinchine formula and is presented in the following theorem (see e.g. [1, Corollary IX.3.4], [23, Corollary 4.9], or [11, 20, 36]).

Theorem 2.1 Consider a Lévy process without negative jumps and with negative drift, i.e., $0<\phi^{\prime}(0)<\infty$. Then, for $\alpha \geq 0$,

$$
\mathbb{E}^{-\alpha Y}=\phi^{\prime}(0) \frac{\alpha}{\phi(\alpha)} .
$$


The distribution of the steady-state workload may be presented in terms of the scale function. In particular, using Definition 2.1 and partial integration, we obtain

$$
\frac{\alpha}{\phi(\alpha)}=\alpha \int_{0}^{\infty} \mathrm{e}^{-\alpha x} W(x) \mathrm{d} x=W(0)+\int_{0^{+}}^{\infty} \mathrm{e}^{-\alpha x} \mathrm{~d} W(x) .
$$

Hence, the transform in Theorem 2.1 may be readily inverted, providing

$$
\mathbb{P}(Y \leq x)=\phi^{\prime}(0) W(x) .
$$

Remark 2.1 The result (2) can also be obtained from the equivalence between the tail distributions of the steady-state workload and ruin probabilities of the time-reversed process, see e.g. [32, Example 1]. From [9, Lemma II.2] or [23, Lemma 3.4] it follows that the time-reversed process and the dual process $\widehat{X}$ have the same law. Starting the dual process at $x$ (thus $X(0)=-x$ ), the ruin probability of $\widehat{X}$ is given in, e.g., [24] by $\mathbb{P}_{-x}\left(T_{0}<\infty\right)=1-\phi^{\prime}(0) W(x)$, assuming that $\phi^{\prime}(0)>0$. Hence, combining these arguments yields

$$
\mathbb{P}(Y \leq x)=1-\mathbb{P}_{-x}\left(T_{0}<\infty\right)=\phi^{\prime}(0) W(x),
$$

corresponding to (2).

In fact, a similar result holds in the case that there is reflection at both 0 and some level $K>0$. We refer to [22] for the formal construction of this process. In a queueing setting, this doubly reflected process is often referred to as the finite dam. We note that reflection at $K$ implies that arriving 'customers' are only partly accepted so that the workload equals $K$. In a queueing situation, there is a well-known proportionality relation between the finite dam and its infinite buffer counterpart. Essentially, this proportionality is the result of the lack-of-memory at downcrossings of $K$ in the infinite buffer queue, such that the sample paths of the finite and infinite buffer queues are identical on $[0, K]$.

Not surprisingly, a similar proportionality result as for the finite dam holds for doubly reflected Lévy processes. Let $Y^{K}$ denote the steady-state workload. The following theorem seems due to [28].

Theorem 2.2 Consider a Lévy process without negative jumps and two-sided reflection. Then, for $x \in[0, K]$,

$$
\mathbb{P}\left(Y^{K} \leq x\right)=\frac{W(x)}{W(K)}
$$

We give here a different proof of Theorem 2.2 based on a duality result of [1, Proposition 3.7]. Another proof of Theorem 2.2, which might be of independent interest, based on the Kella-Whitt martingale can be found in Appendix A.

Proof based on [1] Using the duality between the steady-state versions of reflected processes and exit positions of a dual process with absorbing barriers, specifically [1, 
Proposition 3.7], we have

$$
\mathbb{P}\left(Y^{K}>x\right)=\mathbb{P}_{K-x}\left(T_{K}<\widehat{T}_{0}\right)=1-\frac{W(x)}{W(K)},
$$

where the second equality follows from (1). This completes the proof.

In the notation we add an index $i$ if quantities are associated with stage $i, i=1,2$, e.g. we write $\phi_{i}(\cdot)$ and $W_{i}(\cdot)$.

\section{Analysis of the steady-state workload}

Let $X$ be a right-continuous Lévy process without negative jumps. We exclude the case that $X$ is nondecreasing or a negative drift. In this section we consider the LST and distribution of the steady-state amount of work in the system.

The workload process is defined as $Z(t)=Z(0)+X(t)-I(t)$. This workload process is controlled using an $\left(m_{1}, m_{2}\right)$ policy, with $m_{1}>m_{2} \geq 0$, in the following way: assume that $Z(0)<m_{1}$ and define recursively the switch-over times $T_{m_{1}}^{n}:=$ $\inf \left\{t>T_{m_{2}}^{n-1}: Z(t) \geq m_{1}\right\}$ and $T_{m_{2}}^{n}:=\inf \left\{t>T_{m_{1}}^{n}: Z(t)=m_{2}\right\}, n=0,1, \ldots$, with the convention that $T_{m_{2}}^{-1}=0$. During the intervals $\left[T_{m_{2}}^{n-1}, T_{m_{1}}^{n}\right)$ and $\left[T_{m_{1}}^{n}, T_{m_{2}}^{n}\right), n=$ $0,1, \ldots$, the stage of the system is 1 and 2 , respectively. When the system is in stage $i$ the Lévy exponent of the net input process $X$ is $\phi_{i}(\cdot), i=1,2$. We assume that $\phi_{2}^{\prime}(0)>0$ for the system to be stable. Note that the sign of $\phi_{1}^{\prime}(0)$ is irrelevant for stability, since the process can only drift to infinity in stage 2 .

Obviously, the process $\{Z(t), t \geq 0\}$ is a regenerative process with downcrossings of $m_{2}$ while the system is in stage 2 , i.e., $T_{m_{2}}^{n}, n=0,1, \ldots$, as regeneration points. We may thus consider a single regeneration cycle and distinguish two successive time intervals. More specifically, assume that $Z(0)=m_{2}$ and let $T_{m_{1}}=\inf \{t \geq 0$ : $\left.Z(t) \geq m_{1}\right\}$ and $T_{m_{2}}=\inf \left\{t>T_{m_{1}}: Z(t)=m_{2}\right\}$. During the first interval $\left[0, T_{m_{1}}\right)$ the Lévy exponent equals $\phi_{1}(\cdot)$, while the Lévy exponent equals $\phi_{2}(\cdot)$ during the second interval.

Let $J(t)$ denote the stage of the system and let $Z$ and $J$ denote the steady-state versions of $Z(t)$ and $J(t)$. Using the theory of regenerative processes, we have

$$
\mathbb{E}\left[\mathrm{e}^{-\alpha Z} \mathbf{1}(J=1)\right]=\frac{\mathbb{E}\left[\int_{0}^{T_{m_{1}}} \mathrm{e}^{-\alpha Z(s)} \mathrm{d} s\right]}{\mathbb{E} T_{m_{2}}}
$$

and

$$
\mathbb{E}\left[\mathrm{e}^{-\alpha Z} \mathbf{1}(J=2)\right]=\frac{\mathbb{E}\left[\int_{T_{m_{1}}}^{T_{m_{2}}} \mathrm{e}^{-\alpha Z(s)} \mathrm{d} s\right]}{\mathbb{E} T_{m_{2}}},
$$

where $\mathbf{1}(\cdot)$ is the indicator function. Define for $x \geq 0$ and $i=1,2$,

$$
F_{i}(x)=\mathbb{P}(Z \leq x, J=i) .
$$


3.1 The first interval: $\left[0, T_{m_{1}}\right)$

To determine the steady-state workload, we use the following martingale [1, 20, 23], for $i=1,2$ :

$$
M_{i}(t)=\phi_{i}(\alpha) \int_{s=0}^{t} \mathrm{e}^{-\alpha Z(s)} \mathrm{d} s-\mathrm{e}^{-\alpha Z(t)}+\mathrm{e}^{-\alpha Z(0)}-\alpha I(t) .
$$

For the first interval, take $Z(0)=m_{2}$ and $i=1$. Application of the optional sampling theorem, with stopping time $T_{m_{1}}$ yields (cf. [1, 20]):

$$
\phi_{1}(\alpha) \mathbb{E}\left[\int_{s=0}^{T_{m_{1}}} \mathrm{e}^{-\alpha Z(s)} \mathrm{d} s\right]=\mathbb{E} \mathrm{e}^{-\alpha Z\left(T_{m_{1}}\right)}-\mathrm{e}^{-\alpha m_{2}}+\alpha \mathbb{E} I\left(T_{m_{1}}\right),
$$

or

$$
\mathbb{E}\left[\int_{s=0}^{T_{m_{1}}} \mathrm{e}^{-\alpha Z(s)} \mathrm{d} s\right]=\frac{\mathbb{E} \mathrm{e}^{-\alpha Z\left(T_{m_{1}}\right)}-\mathrm{e}^{-\alpha m_{2}}+\alpha \mathbb{E} I\left(T_{m_{1}}\right)}{\phi_{1}(\alpha)} .
$$

Notice that this expression, when divided by $\mathbb{E} T_{m_{1}}$ (which can be obtained by letting $\alpha \downarrow 0$ in (5)), represents the LST of the steady-state distribution of the $Z$-process given that the stage of the system is 1 .

The transform of the overshoot can be directly obtained from Corollary 2.1 by taking $a=m_{1}$ and $z=m_{2}$, providing, for $\alpha>0$,

$$
\begin{aligned}
\mathbb{E}\left[\mathrm{e}^{-\alpha Z\left(T_{m_{1}}\right)}\right]= & \mathrm{e}^{-\alpha m_{2}}\left(1-\phi_{1}(\alpha) \int_{0}^{m_{1}-m_{2}} \mathrm{e}^{-\alpha y} W_{1}(y) \mathrm{d} y\right) \\
& -\frac{W_{1}\left(m_{1}-m_{2}\right)}{W_{1}^{\prime}\left(m_{1}\right)}\left(\alpha-\phi_{1}(\alpha) \int_{0}^{m_{1}} \mathrm{e}^{-\alpha y} \mathrm{~d} W_{1}(y)\right) .
\end{aligned}
$$

To obtain the constant $\mathbb{E} Z\left(T_{m_{1}}\right)$ we differentiate the above formula with respect to $\alpha$ and let $\alpha \downarrow 0$, yielding

$$
\begin{aligned}
\mathbb{E} Z\left(T_{m_{1}}\right)= & m_{2}+\phi_{1}^{\prime}(0) \int_{0}^{m_{1}-m_{2}} W_{1}(y) \mathrm{d} y \\
& +\frac{W_{1}\left(m_{1}-m_{2}\right)}{W_{1}^{\prime}\left(m_{1}\right)}\left(1-\phi_{1}^{\prime}(0) \int_{0}^{m_{1}} \mathrm{~d} W_{1}(y)\right) .
\end{aligned}
$$

It remains to find the two constants $\mathbb{E} T_{m_{1}}$ and $\mathbb{E} I\left(T_{m_{1}}\right)$. Letting $\alpha \downarrow 0$ in (5) and applying l'Hôpital's rule gives the following relation between $\mathbb{E} T_{m_{1}}$ and $\mathbb{E} I\left(T_{m_{1}}\right)$ :

$$
\phi_{1}^{\prime}(0) \mathbb{E} T_{m_{1}}=m_{2}-\mathbb{E} Z\left(T_{m_{1}}\right)+\mathbb{E} I\left(T_{m_{1}}\right) .
$$

For $\phi^{\prime}(0)=0$, we directly obtain $\mathbb{E} I\left(T_{m_{1}}\right)=\mathbb{E} Z\left(T_{m_{1}}\right)-m_{2}$. In that case, letting $\alpha \downarrow 0$ in (5) and applying l'Hôpital's rule twice gives the constant $\mathbb{E} T_{m_{1}}=$ $\left(\mathbb{E} Z\left(T_{m_{1}}\right)^{2}-m_{2}^{2}\right) / \phi_{1}^{\prime \prime}(0)$. In the case that $\phi_{1}^{\prime}(0) \neq 0$ and $\phi_{1}(\alpha)$ has a non-zero root, say $\hat{\alpha}$, the constant $\mathbb{E} I\left(T_{m_{1}}\right)$ can be directly determined from this non-zero root. Since the expectation in the lhs of (4) is then finite for $\alpha=\hat{\alpha}$, the rhs of (4) should be zero 
for this value of $\alpha$. (We refer to [2] for extending (4) to negative values of $\alpha$; in particular, see [1, Corollary IX.3.7] or [2, Corollary 2] for its direct application in determining the constant.) Hence,

$$
\mathbb{E} I\left(T_{m_{1}}\right)=\frac{1}{\hat{\alpha}}\left(\mathrm{e}^{-\hat{\alpha} m_{2}}-\mathbb{E} \mathrm{e}^{-\hat{\alpha} Z\left(T_{m_{1}}\right)}\right) .
$$

Combining the above gives, for $\phi^{\prime}(0) \neq 0$,

$$
\mathbb{E} T_{m_{1}}=\frac{1}{\phi_{1}^{\prime}(0)}\left(\frac{\mathrm{e}^{-\hat{\alpha} m_{2}}-\mathbb{E} \mathrm{e}^{-\hat{\alpha} Z\left(T_{m_{1}}\right)}}{\hat{\alpha}}-\left(\mathbb{E} Z\left(T_{m_{1}}\right)-m_{2}\right)\right) .
$$

We note that $\phi_{1}(\alpha)$ has a non-zero root in the case $\phi_{1}^{\prime}(0)<0$ (take $\hat{\alpha}=\Phi_{1}(0)>$ 0 ) or, in the case $\phi_{1}^{\prime}(0)>0, \phi_{1}(\cdot)$ has a sufficiently nice structure (see [21] for an example of the superposition of Brownian motion with a compound Poisson process with phase-type jumps). In the case that $\phi_{1}(\alpha)$ only has a zero root, the final constant can be determined by using the first-exit time results of Avram et al. [4] or by first applying Laplace inversion. We refer to (15) and (16) below for the results. This completes the analysis of the LST of $Z$ given that the stage is 1 .

We now turn to the distribution of the steady-state workload by combining the transform for the overshoot (6) with (5) and then applying Laplace inversion. Since the distribution function in stage 1 only has mass on $\left[0, m_{1}\right]$, we invert the LST only on the corresponding interval and neglect the terms having mass on $\left[m_{1}, \infty\right)$, as they cancel out. Note that (5) may be equivalently expressed as

$$
\mathbb{E}\left[\int_{s=0}^{T_{m_{1}}} \mathrm{e}^{-\alpha Z(s)} \mathrm{d} s\right]=\frac{\mathbb{E}^{-\alpha Z\left(T_{m_{1}}\right)}-\mathrm{e}^{-\alpha m_{2}}}{\phi_{1}(\alpha)}+\mathbb{E} I\left(T_{m_{1}}\right) \frac{\alpha}{\phi_{1}(\alpha)} .
$$

The second term can be easily inverted using Definition 2.1. Using (6) to rewrite the first term on the rhs of (11) yields

$$
\begin{aligned}
& \frac{\mathbb{E} \mathrm{e}^{-\alpha Z\left(T_{m_{1}}\right)}-\mathrm{e}^{-\alpha m_{2}}}{\phi_{1}(\alpha)} \\
& =-\mathrm{e}^{-\alpha m_{2}} \int_{0}^{m_{1}-m_{2}} \mathrm{e}^{-\alpha y} W_{1}(y) \mathrm{d} y \\
& \quad-\frac{W_{1}\left(m_{1}-m_{2}\right)}{W_{1}^{\prime}\left(m_{1}\right)}\left(\frac{\alpha}{\phi_{1}(\alpha)}-\int_{0}^{m_{1}} \mathrm{e}^{-\alpha y} \mathrm{~d} W_{1}(y)\right) .
\end{aligned}
$$

Note that $\alpha / \phi_{1}(\alpha)-\int_{0}^{m_{1}} \mathrm{e}^{-\alpha y} \mathrm{~d} W_{1}(y)$ corresponds to the LST of a function with mass only on $\left[m_{1}, \infty\right)$, and thus has no mass on $\left[0, m_{1}\right)$. This can be easily seen from the fact that $W_{1}(\cdot)$ is the Laplace inverse of $\alpha / \phi_{1}(\alpha)$ and, hence, the above two terms cancel out on $\left[0, m_{1}\right)$. The term $\mathrm{e}^{-\alpha m_{2}} \int_{0}^{m_{1}-m_{2}} \mathrm{e}^{-\alpha y} W_{1}(y) \mathrm{d} y$ clearly corresponds to the LST of a function that has no mass on $\left[0, m_{2}\right)$. Applying Laplace inversion, the inverse of this term reads $\int_{0}^{x-m_{2}} W_{1}(y) \mathrm{d} y$, for $x \in\left[m_{2}, m_{1}\right]$.

Summarizing, we have, for $x \in\left[0, m_{2}\right)$,

$$
F_{1}(x)=\frac{\mathbb{E} I\left(T_{m_{1}}\right)}{\mathbb{E} T_{m_{2}}} W_{1}(x)
$$


and, for $x \in\left[m_{2}, m_{1}\right)$,

$$
F_{1}(x)=\frac{\mathbb{E} I\left(T_{m_{1}}\right)}{\mathbb{E} T_{m_{2}}} W_{1}(x)-\frac{1}{\mathbb{E} T_{m_{2}}} \int_{0}^{x-m_{2}} W_{1}(y) \mathrm{d} y .
$$

We conclude with determining the constants $\mathbb{E} I\left(T_{m_{1}}\right)$ and $\mathbb{E} T_{m_{1}}$ for the general case, i.e. including the case that $\phi_{1}(\alpha)$ only has a zero root. Note that

$$
F_{1}\left(m_{1}\right)=\mathbb{P}(J=1)=\frac{\mathbb{E} T_{m_{1}}}{\mathbb{E} T_{m_{2}}},
$$

where the second equality follows from regeneration theory. Taking $x=m_{1}$ in (13) and combining with (7), (8) and (14) yields, after some rewriting,

$$
\mathbb{E} T_{m_{1}}=\frac{W_{1}\left(m_{1}\right) W_{1}\left(m_{1}-m_{2}\right)}{W_{1}^{\prime}\left(m_{1}\right)}-\int_{0}^{m_{1}-m_{2}} W_{1}(y) \mathrm{d} y .
$$

The same result can be derived from the LST of the first-exit time given in [4]. Similarly, using (7) and (8) once more, we have

$$
\mathbb{E} I\left(T_{m_{1}}\right)=\frac{W_{1}\left(m_{1}-m_{2}\right)}{W_{1}^{\prime}\left(m_{1}\right)} .
$$

Remark 3.1 Considering the first interval only and letting $m_{2}=0$, the process corresponds to the workload process in a clearing model, see e.g. [18, Sect. 3] or [21]. The steady-state workload distribution in an M/G/1 queue with clearings (also called disasters) can be found in [6], where it follows as a special case. Equation (13) may thus also be considered as an extension of clearing models to spectrally positive Lévy processes.

\subsection{The second interval: $\left[T_{m_{1}}, T_{m_{2}}\right]$}

In this subsection, we consider the second interval. The stage of the system is 2 during this interval and the Lévy exponent thus equals $\phi_{2}(\cdot)$. Note that the process restricted to the second stage can be interpreted as a reflected Lévy process with secondary jump input, see [19]. In particular, as soon as the process hits $m_{2}$ it jumps by a random amount distributed as the overshoot at the end of the first phase. Hence, the steady-state results for the process restricted to stage 2 can be directly obtained from [19]. For later use, in particular in Sects. 4 and 5, we outline its derivation.

An application of the martingale $M_{2}(t)$, now with stopping time $T_{m_{2}}$ and some rewriting, yields

$$
\mathbb{E}\left[\int_{s=T_{m_{1}}}^{T_{m_{2}}} \mathrm{e}^{-\alpha Z(s)} \mathrm{d} s\right]=\frac{\mathrm{e}^{-\alpha m_{2}}-\mathbb{E}\left[\mathrm{e}^{-\alpha Z\left(T_{m_{1}}\right)}\right]}{\phi_{2}(\alpha)},
$$

with $\mathbb{E}\left[\mathrm{e}^{-\alpha Z\left(T_{m_{1}}\right)}\right]$ given by (6). The remaining constant $\mathbb{E}\left[T_{m_{2}}-T_{m_{1}}\right]$ is obtained by letting $\alpha \downarrow 0$ in (17) and applying l'Hôpital's rule. Using (8) (for $\phi_{1}^{\prime}(0) \neq 0$ ), we 
have

$$
\mathbb{E} T_{m_{2}}=\frac{\mathbb{E} I\left(T_{m_{1}}\right)}{\phi_{1}^{\prime}(0)}+\left(\frac{1}{\phi_{1}^{\prime}(0)}-\frac{1}{\phi_{2}^{\prime}(0)}\right)\left(m_{2}-\mathbb{E} Z\left(T_{m_{1}}\right)\right),
$$

with $\mathbb{E} I\left(T_{m_{1}}\right)$ and $\mathbb{E} Z\left(T_{m_{1}}\right)$ given in (16) and (7) respectively. Note that we have completely determined the LST of $Z$, including the constants.

Define the random variable $Z_{m_{2}}^{*}:=Z\left(T_{m_{1}}\right)-m_{2}$ as the overshoot over $m_{2}$ at the end of the first interval, i.e., at time $T_{m_{1}}$. After some basic manipulations, we may derive from (17)

$$
\frac{1}{\mathbb{E}\left[T_{m_{2}}-T_{m_{1}}\right]} \mathbb{E}\left[\int_{s=T_{m_{1}}}^{T_{m_{2}}} \mathrm{e}^{-\alpha Z(s)} \mathrm{d} s\right]=\mathrm{e}^{-\alpha m_{2}} \phi_{2}^{\prime}(0) \frac{\alpha}{\phi_{2}(\alpha)} \frac{1-\mathbb{E}\left[\mathrm{e}^{-\alpha Z_{m_{2}}^{*}}\right]}{\alpha \mathbb{E} Z_{m_{2}}^{*}} .
$$

This term can be easily inverted using the fact that the product of LSTs corresponds to the convolution of random variables, giving the following decomposition result:

$$
(Z \mid J=2)={ }^{d} m_{2}+W_{2}+Z_{m_{2}}^{*, \text { res }}
$$

where $W_{2}$ and $Z_{m_{2}}^{* \text {,res }}$ denote generic random variables for the steady-state workload of a reflected Lévy process with exponent $\phi_{2}(\cdot)$ and the residual overshoot over $m_{2}$ at time $T_{m_{1}}$, respectively. This decomposition result may also follow directly from Theorem 6.1 and Corollary 6.1 in [19].

Finally, we focus on the distribution of $Z_{m_{2}}^{* \text {,res }}$. Using its definition and (6) we obtain, after some rewriting,

$$
\begin{aligned}
\mathbb{E e}^{-\alpha Z_{m_{2}}^{*, \text { res }}}= & \frac{1-\mathrm{e}^{\alpha m_{2}} \mathbb{E}\left[\mathrm{e}^{-\alpha Z\left(T_{m_{1}}\right)}\right]}{\alpha \mathbb{E} Z_{m_{2}}^{*}} \\
= & \frac{1}{\mathbb{E} Z_{m_{2}}^{*}}\left[\frac{\phi_{1}(\alpha)}{\alpha} \int_{0}^{m_{1}-m_{2}} \mathrm{e}^{-\alpha y} W_{1}(y) \mathrm{d} y\right. \\
& \left.+\frac{W_{1}\left(m_{1}-m_{2}\right)}{W_{1}^{\prime}\left(m_{1}\right)} \mathrm{e}^{\alpha m_{2}}\left(1-\frac{\phi_{1}(\alpha)}{\alpha} \int_{0}^{m_{1}} \mathrm{e}^{-\alpha y} \mathrm{~d} W_{1}(y)\right)\right] .
\end{aligned}
$$

The distribution of $Z_{m_{2}}^{* \text {,res }}$ can now be obtained by applying Laplace inversion. However, we characterize the distribution of $Z_{m_{2}}^{* \text {,res }}$ by two implicit equations (see also Remark 3.2 below for another alternative).

Multiplying both sides by $\alpha / \phi_{1}(\alpha)$ yields

$$
\begin{aligned}
\frac{\alpha}{\phi_{1}(\alpha)} \mathbb{E} \mathrm{e}^{-\alpha Z_{m_{2}}^{*, \text { res }}=}= & \frac{1}{\mathbb{E} Z_{m_{2}}^{*}}\left[\int_{0}^{m_{1}-m_{2}} \mathrm{e}^{-\alpha y} W_{1}(y) \mathrm{d} y\right. \\
& \left.+\frac{W_{1}\left(m_{1}-m_{2}\right)}{W_{1}^{\prime}\left(m_{1}\right)} \mathrm{e}^{\alpha m_{2}}\left(\frac{\alpha}{\phi_{1}(\alpha)}-\int_{0}^{m_{1}} \mathrm{e}^{-\alpha y} \mathrm{~d} W_{1}(y)\right)\right] .
\end{aligned}
$$

Note that the inverse of $\alpha / \phi_{1}(\alpha)-\int_{0}^{m_{1}} \mathrm{e}^{-\alpha y} \mathrm{~d} W_{1}(y)$ equals $W_{1}(\cdot)$ on $\left[m_{1}, \infty\right)$ and 0 otherwise. Applying Laplace inversion provides two implicit equations for the distri- 
bution of $Z_{m_{2}}^{* \text {,res }}$ : For $x \in\left[0, m_{1}-m_{2}\right)$, we have

$$
\int_{0}^{x} \mathbb{P}\left(Z_{m_{2}}^{*, \text { res }} \leq x-y\right) \mathrm{d} W_{1}(y)=\frac{1}{\mathbb{E} Z_{m_{2}}^{*}} \int_{0}^{x} W_{1}(y) \mathrm{d} y,
$$

and, for $x \geq m_{1}-m_{2}$,

$$
\begin{aligned}
& \int_{0}^{x} \mathbb{P}\left(Z_{m_{2}}^{*, \text { res }} \leq x-y\right) \mathrm{d} W_{1}(y) \\
& \quad=\frac{1}{\mathbb{E} Z_{m_{2}}^{*}}\left[\frac{W_{1}\left(m_{1}-m_{2}\right)}{W_{1}^{\prime}\left(m_{1}\right)} W_{1}\left(x+m_{2}\right)+\int_{0}^{m_{1}-m_{2}} W_{1}(y) \mathrm{d} y\right] .
\end{aligned}
$$

Remark 3.2 In several special cases it is possible to give explicit expressions for the steady-state workload distribution in stage 2 , i.e. $F_{2}(\cdot)$, depending on the specific form of $\phi_{1}(\alpha) / \phi_{2}(\alpha)$. The cases of a change of drift, Brownian motions, and M/G/1 queues are addressed in Sect. 4.

For this alternative approach, we express the LST of $Z\left(T_{m_{1}}\right)$ in terms of the steadystate workload of the first interval. In particular, we rewrite (4) as

$$
\mathbb{E} \mathrm{e}^{-\alpha Z\left(T_{m_{1}}\right)}=\phi_{1}(\alpha) \mathbb{E}\left[\int_{s=0}^{T_{m_{1}}} \mathrm{e}^{-\alpha Z(s)} \mathrm{d} s\right]+\mathrm{e}^{-\alpha m_{2}}-\alpha \mathbb{E} I\left(T_{m_{1}}\right) .
$$

Substituting the above in (17) yields

$$
\mathbb{E}\left[\int_{s=T_{m_{1}}}^{T_{m_{2}}} \mathrm{e}^{-\alpha Z(s)} \mathrm{d} s\right]=\frac{\alpha}{\phi_{2}(\alpha)} \mathbb{E} I\left(T_{m_{1}}\right)-\frac{\phi_{1}(\alpha)}{\phi_{2}(\alpha)} \mathbb{E}\left[\int_{0}^{T_{m_{1}}} \mathrm{e}^{-\alpha Z(s)} \mathrm{d} s\right] .
$$

The Laplace inversion of the rhs of the above expression is demonstrated in Sect. 4 for various special cases.

\section{Special cases}

In this section, we consider the following three special cases that are of independent interest: (i) Change of drift, (ii) general M/G/1 queues, and (iii) Brownian motion. In these special cases, the steady-state workload distribution in stage 2, i.e. $F_{2}(\cdot)$, has a more tractable form. Moreover, for the latter two special cases the scale function can be explicitly given. We refer to [17, 25] for further examples where the scale function is known in explicit form.

Since an explicit formula for $F_{1}(\cdot)$ is given by (12) and (13), we mainly focus on the second interval. Dividing both sides of (20) by $\mathbb{E} T_{m_{2}}$ and some rewriting provides the following form for the LST of the steady-state workload in stage 2: 


$$
\begin{aligned}
& \frac{1}{\mathbb{E} T_{m_{2}}} \mathbb{E}\left[\int_{s=T_{m_{1}}}^{T_{m_{2}}} \mathrm{e}^{-\alpha Z(s)} \mathrm{d} s\right] \\
& \quad=\frac{\mathbb{E} I\left(T_{m_{1}}\right)}{\mathbb{E} T_{m_{2}}} \frac{\alpha}{\phi_{2}(\alpha)}+\frac{\phi_{2}(\alpha)-\phi_{1}(\alpha)}{\phi_{2}(\alpha)} \frac{\mathbb{E}\left[\int_{0}^{T_{m_{1}}} \mathrm{e}^{-\alpha Z(s)} \mathrm{d} s\right]}{\mathbb{E} T_{m_{2}}}-\frac{\mathbb{E}\left[\int_{0}^{T_{m_{1}}} \mathrm{e}^{-\alpha Z(s)} \mathrm{d} s\right]}{\mathbb{E} T_{m_{2}}} .
\end{aligned}
$$

The first and third terms on the rhs can be readily inverted yielding $W_{2}(x) \mathbb{E} I\left(T_{m_{1}}\right) / \mathbb{E} T_{m_{2}}$ and $-F_{1}(x)$, respectively. For the second term on the rhs of (21), we reduce $\left(\phi_{2}(\alpha)-\phi_{1}(\alpha)\right) / \phi_{2}(\alpha)$ for the special cases mentioned above, see also [8]. Applying Laplace inversion then provides intuitive formulas for $F_{2}(\cdot)$.

Change of drift Here, we consider the appealing special case of a change of drift, i.e., we assume that $\phi_{1}(\alpha)=\left(r_{1}-r_{2}\right) \alpha+\phi_{2}(\alpha)$. This case is most often studied in the literature, usually under the assumption of an M/G/1 setting or for Brownian motion. Note that

$$
\frac{\phi_{2}(\alpha)-\phi_{1}(\alpha)}{\phi_{2}(\alpha)}=\left(r_{2}-r_{1}\right) \frac{\alpha}{\phi_{2}(\alpha)},
$$

where the rhs can be easily inverted using the scale function $W_{2}(\cdot)$. Using the fact that the product of two LSTs corresponds to the convolution of two functions, we obtain, by applying Laplace inversion to (21), for $x \geq m_{2}$,

$$
F_{2}(x)=\frac{\mathbb{E} I\left(T_{m_{1}}\right)}{\mathbb{E} T_{m_{2}}} W_{2}(x)+\left(r_{2}-r_{1}\right) \int_{0}^{x} W_{2}(x-y) \mathrm{d} F_{1}(y)-F_{1}(x),
$$

with $F_{1}(\cdot)$ given by (12) and (13).

$M / G / 1$ queue Consider the general M/G/1 case with arrival rates $\lambda_{i}$, service speeds $r_{i}$, and generic service requirements $B_{i}$ with distribution $B_{i}(\cdot)$, mean $\beta_{i}$, and LST $\beta_{i}(\cdot), i=1,2$. Note that $\phi_{i}(\alpha)=r_{i} \alpha-\lambda_{i}+\lambda_{i} \beta_{i}(\alpha)$ in this case.

For M/G/1 queues, the scale function has a tractable form. Let $\rho_{i}:=\lambda_{i} \beta_{i} / r_{i}, i=$ 1,2 , denote the traffic intensity in stage $i$ and let $H_{i}(x):=\beta_{i}^{-1} \int_{0}^{x}\left(1-B_{i}(y)\right)$ dy be the stationary residual service requirement distribution. The stability condition now reads $\rho_{2}<1$. In the case $\rho_{i}<1$, it is well known that

$$
W_{i}(x)=\frac{1}{r_{i}} \sum_{n=0}^{\infty} \rho_{i}^{n} H_{i}^{n^{*}}(x),
$$

where $H_{i}^{n^{*}}(\cdot)$ denotes the $n$-fold convolution of $H_{i}(\cdot)$ with itself. The steadystate workload distribution for an $\mathrm{M} / \mathrm{G} / 1$ queue with exponent $\phi_{i}(\cdot)$ then reads $\left(1-\rho_{i}\right) r_{i} W_{i}(x)$. For the case $\rho_{1}>1$, we refer to e.g. [8] for details.

Also, the distribution function on the second interval can be rewritten using (21). After some basic algebra, we can rewrite the fraction of Lévy exponents into familiar terms:

$$
\frac{\phi_{2}(\alpha)-\phi_{1}(\alpha)}{\phi_{2}(\alpha)}=-r_{1} \frac{\alpha}{r_{2} \alpha-\lambda_{2}+\lambda_{2} \beta_{2}(\alpha)}+\frac{\lambda_{1}}{\lambda_{2}} \frac{r_{2} \alpha-\lambda_{2} \beta_{1}(\alpha)+\lambda_{2} \beta_{2}(\alpha)}{r_{2} \alpha-\lambda_{2}+\lambda_{2} \beta_{2}(\alpha)}+1-\frac{\lambda_{1}}{\lambda_{2}} .
$$


Observe that the first term on the rhs is related to the familiar M/G/1 queue and the second term is related to an $\mathrm{M} / \mathrm{G} / 1$ queue with an exceptional first service time. Let $W_{2}^{\text {exc }}(\cdot)$ be the steady-state workload distribution in an $\mathrm{M} / \mathrm{G} / 1$ queue with service rate $r_{2}$, arrival rate $\lambda_{2}$, and generic service requirement $B_{2}$, but with exceptional first service $B_{1}$ in a busy period (see e.g. [33], p. 128 or [8, Example A.1]). Combining the above with (21) and applying Laplace inversion yields, for $x \geq m_{2}$,

$$
\begin{aligned}
F_{2}(x)= & \frac{\mathbb{E} I\left(T_{m_{1}}\right)}{\mathbb{E} T_{m_{2}}} W_{2}(x)-r_{1} \int_{0}^{x} W_{2}(x-y) \mathrm{d} F_{1}(y) \\
& +\frac{\lambda_{1}}{\lambda_{2}} \frac{1+\frac{\lambda_{2} \beta_{1}}{r_{2}}-\rho_{2}}{1-\rho_{2}} \int_{0}^{x} W_{2}^{\operatorname{exc}}(x-y) \mathrm{d} F_{1}(y)-\frac{\lambda_{1}}{\lambda_{2}} F_{1}(x),
\end{aligned}
$$

with $F_{1}(\cdot)$ given by (12) and (13).

Brownian motion In the case of Brownian motion, we assume that $\phi_{i}(\alpha)=$ $\alpha^{2} \sigma_{i}^{2} / 2-\mu_{i} \alpha, i=1,2$. Hence, the scale function reads $W_{i}(x)=\left(\exp \left(2 \mu_{i} x / \sigma_{i}^{2}\right)-\right.$ $1) / \mu_{i}$. For abbreviation, we denote $\eta_{i}=2 \mu_{i} / \sigma_{i}^{2}$. Note that, for $\mu_{1} \neq 0, \phi_{1}(\alpha)=0$ has a unique non-zero solution $\hat{\alpha}=\eta_{1}$. Since $Z\left(T_{m_{1}}\right)=m_{1}$, using (9) and (18), we obtain the constants $\mathbb{E} I\left(T_{m_{1}}\right)=\left(\exp \left(-\eta_{1} m_{2}\right)-\exp \left(-\eta_{1} m_{1}\right)\right) / \eta_{1}$ and

$$
\mathbb{E} T_{m_{2}}=\left(\frac{1}{\mu_{1}}-\frac{1}{\mu_{2}}\right)\left(m_{1}-m_{2}\right)-\frac{1}{\mu_{1} \eta_{1}}\left(\mathrm{e}^{-\eta_{1} m_{2}}-\mathrm{e}^{-\eta_{1} m_{1}}\right) .
$$

Substitution in (12) and (13) yields, for $x \in\left[0, m_{2}\right)$,

$$
F_{1}(x)=\frac{\mathbb{E} I\left(T_{m_{1}}\right)}{\mu_{1} \mathbb{E} T_{m_{2}}}\left(\mathrm{e}^{\eta_{1} x}-1\right),
$$

and, using the specific form of $\mathbb{E} I\left(T_{m_{1}}\right)$, for $x \in\left[m_{2}, m_{1}\right)$,

$$
F_{1}(x)=\frac{1}{\mu_{1} \mathbb{E} T_{m_{2}}}\left[\frac{1}{\eta_{1}}\left(1-\mathrm{e}^{\eta_{1}\left(x-m_{1}\right)}\right)+\frac{1}{\eta_{1}}\left(\mathrm{e}^{-\eta_{1} m_{1}}-\mathrm{e}^{-\eta_{1} m_{2}}\right)+x-m_{2}\right] .
$$

For the second interval, we use the analysis of Sect. 3.2. Because Brownian motion has continuous sample paths, we have a deterministic overshoot $Z_{m_{2}}^{*}=m_{1}-m_{2}$. Consequently, the residual overshoot $Z_{m_{2}}^{* \text {,res }}$ is uniformly distributed on the interval $\left[m_{2}, m_{1}\right]$. Applying the decomposition result, we find after some straightforward computations that, for $x \in\left[m_{2}, m_{1}\right)$,

$$
F_{2}(x)=\frac{1}{\mu_{2} \mathbb{E} T_{m_{2}}}\left[\frac{1}{\eta_{2}}\left(\mathrm{e}^{\eta_{2}\left(x-m_{2}\right)}-1\right)-\left(x-m_{2}\right)\right],
$$

and for $x \geq m_{1}$,

$$
F_{2}(x)=\frac{1}{\mu_{2} \mathbb{E} T_{m_{2}}}\left[\frac{1}{\eta_{2}} \mathrm{e}^{\eta_{2}\left(x-m_{1}\right)}\left(\mathrm{e}^{\eta_{2}\left(m_{1}-m_{2}\right)}-1\right)-\left(m_{1}-m_{2}\right)\right] .
$$




\section{Doubly reflected process}

In this section we assume that the workload process is also reflected at some level $K \geq m_{1}$, representing a finite buffer capacity. Such finite capacity systems are well known in queueing scenarios and also often appear in systems with hysteretic control.

Since the first interval ends as soon as the workload upcrosses level $m_{1} \leq K$, the reflection only effects the workload process on the interval $\left[0, T_{m_{1}}\right]$ through the constant $\mathbb{E} T_{m_{2}}$, see (26) below. This constant involves the only adaptation in the steady-state results for this interval, i.e. $F_{1}(\cdot)$. To analyze the steady-state workload distribution during the second interval, we first use martingale arguments [1, 20] again to obtain the LST. Since the workload process is kept in $\left[m_{2}, K\right]$, the workload distribution has no mass on $[K, \infty)$. Hence, we invert the LST on the interval $\left[m_{2}, K\right]$ to find the steady-state amount of work. The terms where the inverse has mass on $[K, \infty)$ can be neglected, because they cancel out.

More precisely, during the second interval, the workload process is defined as $Z^{K}(t)=Z_{0}^{*}+X\left(t-T_{m_{1}}\right)-S^{K}(t)$, where $Z_{0}^{*}=Z\left(T_{m_{1}}\right)$ and $S^{K}(t):=$ $\sup _{T_{m_{1} \leq s \leq t}}\left\{\left(X\left(s-T_{m_{1}}\right)+Z_{0}^{*}-K\right) \vee 0\right\}$, for $t \in\left[T_{m_{1}}, T_{m_{2}}^{K}\right]$ with $T_{m_{2}}^{K}:=\inf \{t \geq$ $\left.T_{m_{1}}: Z^{K}(t)=m_{2}\right\}$ (see [22]). Here $X$ is a Lévy process with exponent $\phi_{2}(\cdot)$. Notice that there is no reflection term in 0 , because the horizontal axis is not hit during this second interval. Also, the local time at $K, S^{K}(t)$, is an adapted càdlàg process. Because $S^{K}(t)$ is increasing, it is of bounded variation on finite intervals. We decompose the local time at $K$ into its continuous and its jump part (see e.g. [3, 20], or [9], p. 208)

$$
S^{K}(t)=S^{K, c}(t)+\sum_{T_{m_{1}} \leq s \leq t} \Delta S^{K}(s),
$$

where $\Delta S^{K}(t)=S^{K}(t)-S^{K}\left(t^{-}\right)$with $S^{K}\left(t^{-}\right)=\lim _{s \uparrow t} S^{K}(s)$.

We now apply the martingale used in [1, 20, 23] or, more specifically, [3, Proposition 3.3]:

$$
\begin{aligned}
M^{K}(t)= & \phi_{2}(\alpha) \int_{T_{m_{1}}}^{t} \mathrm{e}^{-\alpha Z^{K}(s)} \mathrm{d} s-\mathrm{e}^{-\alpha Z^{K}(t)}+\mathrm{e}^{-\alpha Z_{0}^{*}} \\
& +\alpha \mathrm{e}^{-\alpha K} S^{K, c}(t)+\mathrm{e}^{-\alpha K} \sum_{T_{m_{1}} \leq s \leq t}\left(1-\mathrm{e}^{-\alpha \Delta S^{K}(s)}\right) .
\end{aligned}
$$

Using the definition of $Z_{m_{2}}^{*}$ it follows from an application of the martingale $M^{K}(t)$ with stopping time $T_{m_{2}}^{K}$, in addition to some rewriting, that 


$$
\begin{aligned}
\mathbb{E}\left[\int_{s=T_{m_{1}}}^{T_{m_{2}}^{K}} \mathrm{e}^{-\alpha Z^{K}(s)} \mathrm{d} s\right] \\
=\mathbb{E} Z_{m_{2}}^{*} \frac{\alpha}{\phi_{2}(\alpha)} \mathrm{e}^{-\alpha m_{2}} \frac{1-\mathbb{E}\left[\mathrm{e}^{\left.-\alpha Z_{m_{2}}^{*}\right]}\right.}{\alpha \mathbb{E} Z_{m_{2}}^{*}}-\frac{\alpha}{\phi_{2}(\alpha)} \mathrm{e}^{-\alpha K} \mathbb{E} S^{K, c}\left(T_{m_{2}}^{K}\right) \\
\quad-\frac{\alpha}{\phi_{2}(\alpha)} \mathrm{e}^{-\alpha K} \mathbb{E}\left[\sum_{T_{m_{1}} \leq s \leq T_{m_{2}}^{K}} \frac{1-\mathrm{e}^{-\alpha \Delta S^{K}(s)}}{\alpha}\right] .
\end{aligned}
$$

The above expression provides the steady-state workload during the second interval, up to a constant. Because the workload process is constructed such that $Z^{K}(t) \in\left[m_{2}, K\right]$, we only need to invert each of the three terms on the rhs of the above equation on the corresponding interval. Neglecting terms with mass on $[K, \infty)$ greatly simplifies the Laplace inversion. (Note again that the terms with mass on $[K, \infty)$ should cancel out.)

To control the final term on the rhs of (23), we need the following lemma:

Lemma 5.1 The term $\mathbb{E}\left[\sum_{T_{m_{1}} \leq s \leq T_{m_{2}}^{K}} \frac{1-\mathrm{e}^{-\alpha \Delta S^{K}(s)}}{\alpha}\right]$ corresponds to the LST of a function with no mass on $(-\infty, 0)$.

Proof Let $\tau_{K}:=\inf \left\{t \geq T_{m_{1}}: Z^{K}(t)=K\right\}$ be the first hitting time of $K$. Note that $\tau_{K}$ can be equal to $T_{m_{1}}$, in which case $S^{K}\left(T_{m_{1}}\right)=Z_{0}^{*}-K$.

In the case $\tau_{K}>T_{m_{2}}$, the process does not hit $K$ and the lemma is trivial. For the case $\tau_{K}<T_{m_{2}}$ observe that $S^{K}\left(\tau_{K}+t\right)-S^{K}\left(\tau_{K}\right)$ has the same law as the supremum process $S(t):=\sup _{0 \leq s \leq t}\{X(s) \vee 0\}$ and $Z^{K}\left(\tau_{K}+t\right)$ has the same law as $X-S+K$ until $X-S+K \leq m_{2}$. Define $\tau_{m_{2}}:=\inf \left\{t \geq 0: X(t)-S(t)+K=m_{2}\right\}$. As in e.g. $[9,23]$, denote by $L=\{L(t), t \geq 0\}$ the local time of $S-X$ at 0 and by $L^{-1}(t)=$ $\inf \{s>0: L(s)>t\}$ its right-continuous inverse. We also introduce the ladder height process $H$, using the inverse local time to time-change the supremum process, as $H(t)=S\left(L^{-1}(t)\right)$, see [9], p. 157, or [23], p. 147. Since the jumps in the supremum process correspond to jumps in the ladder height process, we have

$$
\begin{aligned}
& \mathbb{E}\left[\sum_{T_{m_{1}} \leq s \leq T_{m_{2}}^{K}} \frac{1}{\alpha}\left(1-\mathrm{e}^{-\alpha \Delta S^{K}(s)}\right)\right] \\
& \quad=\mathbb{P}\left(\tau_{K}<T_{m_{2}}\right)\left(\frac{1}{\alpha} \mathbb{E}\left[1-\mathrm{e}^{-\alpha \Delta S^{K}\left(\tau_{K}\right)}\right]+\frac{1}{\alpha} \mathbb{E}\left[\sum_{\tau_{K} \leq s \leq T_{m_{2}}^{K}}\left(1-\mathrm{e}^{-\alpha \Delta S^{K}(s)}\right)\right]\right) \\
& \quad=\mathbb{P}\left(\tau_{K}<T_{m_{2}}\right)\left(\frac{1}{\alpha} \mathbb{E}\left[1-\mathrm{e}^{-\alpha \Delta S^{K}\left(\tau_{K}\right)}\right]+\frac{1}{\alpha} \mathbb{E}\left[\sum_{0 \leq s \leq L\left(\tau_{m_{2}}\right)}\left(1-\mathrm{e}^{-\alpha \Delta H\left(L^{-1}(s)\right)}\right)\right]\right) .
\end{aligned}
$$

For the first term $\left(1-\mathbb{E} \mathrm{e}^{-\alpha \Delta S^{K}\left(\tau_{K}\right)}\right) / \alpha$ we observe that it corresponds to the tail of the overshoot over $K$ at the first-exit time of $[0, K]$. Hence, this is a transform of 
a function with mass only on $[0, \infty)$. For the second term, we use some properties of the ladder height process $H$. In particular, we use the well-known result that the ladder height process is a (nondecreasing) Lévy process, see e.g. [9, Lemma VI.2]. Let the Lévy measure of $H$ be given by $v_{H}(\cdot)$.

Using the compensation formula for Poisson point processes, we obtain for the second term

$$
\begin{aligned}
& \frac{1}{\alpha} \mathbb{E}\left[\sum_{0 \leq s \leq L\left(\tau_{m_{2}}\right)}\left(1-\mathrm{e}^{-\alpha \Delta H\left(L^{-1}(s)\right)}\right)\right] \\
& =\mathbb{E}\left[\int_{0}^{\infty} \mathbf{1}\left(t \leq L\left(\tau_{m_{2}}\right)\right) \mathrm{d} t \int_{0}^{\infty} \frac{1-\mathrm{e}^{-\alpha s}}{\alpha} v_{H}(\mathrm{~d} s)\right] \\
& =\mathbb{E} L\left(\tau_{m_{2}}\right) \int_{0}^{\infty} \mathrm{e}^{-\alpha s} v_{H}((s, \infty)) \mathrm{d} s .
\end{aligned}
$$

Note that $\int_{0}^{\infty} \mathrm{e}^{-\alpha s} v_{H}((s, \infty)) \mathrm{d} s$ is the LST of the tail of the Lévy measure of $H$. Clearly, the tail $v_{H}((\cdot, \infty))$ has no mass on $(-\infty, 0)$, which completes the proof.

Remark 5.1 In the case that the process with exponent $\phi_{2}(\cdot)$ is of bounded variation, Lemma 5.1 can be derived using direct probabilistic arguments. Note that the probability that the process hits level $m_{2}$ before level $K$ when the initial position is $K$ equals $W(0) / W\left(K-m_{2}\right)$, cf. (1). This probability is strictly positive for processes of bounded variation, see e.g. [4]. Hence, given that the process $X$ first upcrosses level $K$, the number of successive upcrossings of level $K$ before the first downcrossing of $m_{2}$ is geometrically distributed with parameter $W(0) / W\left(K-m_{2}\right)>0$. The second term in (24) can then be directly treated using Wald's equation, giving a geometric number of residual overshoots. This clearly has no mass on $(-\infty, 0)$.

Now, we are ready to invert each of the terms on the rhs of (23) separately on the interval $\left[m_{2}, K\right]$. The Laplace inversion of the first term is similar to the case of an infinite buffer, see (19). In particular, the inverse of $\alpha / \phi_{2}(\alpha)$ is given by $W_{2}(\cdot)$ and $\left(1-\mathbb{E}\left[\mathrm{e}^{-\alpha Z_{m_{2}}^{*}}\right]\right) /\left(\alpha \mathbb{E} Z_{m_{2}}^{*}\right)$ corresponds to the LST of $Z_{m_{2}}^{* \text {,res }}$. The product of these transforms thus represents the convolution $W_{2}(x) * \mathbb{P}\left(Z_{m_{2}}^{* \text {,res }} \leq x\right)$, where $*$ denotes a convolution.

We note that the second and third terms on the rhs of (23) only have mass on $[K, \infty)$. To see this, note that $\alpha / \phi_{2}(\alpha)$ is the LST of $W_{2}(\cdot)$ and has mass on $[0, \infty)$. Consequently, $\mathrm{e}^{-\alpha K} \alpha / \phi_{2}(\alpha)$ is the transform of a function with mass on $[K, \infty)$. This completes the second term.

The third term involves the product of two transforms, corresponding to the convolution of two functions. Since $\mathrm{e}^{-\alpha K} \alpha / \phi_{2}(\alpha)$ only has mass on $[K, \infty)$, it follows from Lemma 5.1 that the third term also only has mass on $[K, \infty)$. Hence, for $x \in\left[0, K-m_{2}\right]$, we have

$$
\mathbb{P}\left(Z^{K} \leq x+m_{2}, J=2\right)=\frac{\mathbb{E} Z\left(T_{m_{1}}\right)-m_{2}}{\mathbb{E} T_{m_{2}}} \int_{0}^{x} W_{2}(x-y) \mathrm{d} \mathbb{P}\left(Z_{m_{2}}^{*, \text { res }} \leq y\right) .
$$


Finally, it remains to find the normalizing constant $\mathbb{E} T_{m_{2}}$. From regeneration theory it follows again that

$$
\mathbb{P}\left(Z^{K} \leq K, J=2\right)=\mathbb{P}(J=2)=1-\frac{\mathbb{E} T_{m_{1}}}{\mathbb{E} T_{m_{2}}}
$$

Using the above and taking $x=K-m_{2}$ in (25), yields

$$
\mathbb{E} T_{m_{2}}=\mathbb{E} T_{m_{1}}+\left(\mathbb{E} Z\left(T_{m_{1}}\right)-m_{2}\right) \int_{0}^{K-m_{2}} W_{2}\left(K-m_{2}-y\right) \mathrm{d} \mathbb{P}\left(Z_{m_{2}}^{*, \mathrm{res}} \leq y\right) \text {. }
$$

Corollary 5.1 For $\phi_{2}^{\prime}(0)>0$ and $x \in\left[m_{2}, K\right]$, we have

$$
\mathbb{P}\left(Z^{K} \leq x \mid J=2\right)=\frac{\mathbb{P}(Z \leq x \mid J=2)}{\mathbb{P}(Z \leq K \mid J=2)} .
$$

The results for the various special cases of Sect. 4 can also be easily modified in case of a finite buffer. In particular, Corollary 5.1 shows that the steady-state workload distribution in the case of finite buffers (and $\phi_{2}^{\prime}(0)>0$ ) is just the truncated workload distribution of the case of infinite buffers.

An alternative proof of Theorem 2.2 using similar arguments as above, in particular (22) and Lemma 5.1, is given in Appendix A.

Acknowledgements The author is grateful to two anonymous referees for their valuable comments.

Open Access This article is distributed under the terms of the Creative Commons Attribution Noncommercial License which permits any noncommercial use, distribution, and reproduction in any medium, provided the original author(s) and source are credited.

\section{Appendix A: Martingale proof of Theorem 2.2}

Here, we give an alternative proof of Theorem 2.2 based on martingales and Laplace inversion on $[0, K]$. The analysis is similar to the derivation in Sect. 5. In principle, letting $\phi_{1}(\alpha) \equiv \phi_{2}(\alpha) \equiv \phi(\alpha)$, the proportionality result can also be derived from the results in that section. However, we believe that this alternative derivation of Theorem 2.2 might be of independent interest giving insights for many finite-buffer systems.

Due to similarities with Sect. 5, we continue to use the notation $\left\{Z^{K}(t), t \geq 0\right\}$ for the doubly reflected process. However, we denote the steady-state amount of work in the system by $Y^{K}$, see also Sect. 2 .

Alternative proof of Theorem 2.2 Using the first-hitting time definitions of Sect. 3 with $m_{1}=K$ and $m_{2}=0$ it holds that $T_{0}^{n}, n=0,1, \ldots$, are regeneration epochs. A regenerative cycle thus starts at time 0 with $Z^{K}(0)=0$ and ends when the process hits 0 after first upcrossing level $K$ (the cycle length is denoted by $T_{0}$ ). 
We let $I^{K}=\left\{I^{K}(t), t \geq 0\right\}$ be the local time at 0 of the doubly reflected process. Note that for $t \in\left[0, T_{0}\right)$ it holds that $I(t)=I^{K}(t)$. We use the following martingale (see $[1,20,23]$ or [3, Proposition 3.3]):

$$
\begin{aligned}
M^{K}(t)= & \phi(\alpha) \int_{0}^{t} \mathrm{e}^{-\alpha Z^{K}(s)} \mathrm{d} s-\mathrm{e}^{-\alpha Z^{K}(t)}+\mathrm{e}^{-\alpha Z^{K}(0)}-\alpha I^{K}(t) \\
& +\alpha \mathrm{e}^{-\alpha K} S^{K, c}(t)+\mathrm{e}^{-\alpha K} \sum_{0 \leq s \leq t}\left(1-\mathrm{e}^{-\alpha \Delta S^{K}(s)}\right) .
\end{aligned}
$$

Applying the optional sampling theorem, with stopping time $T_{0}$, we obtain

$$
\begin{aligned}
\mathbb{E}\left[\int_{s=0}^{T_{0}} \mathrm{e}^{-\alpha Z^{K}(s)} \mathrm{d} s\right]= & \frac{\alpha}{\phi(\alpha)} \mathbb{E} I^{K}\left(T_{0}\right)-\frac{\alpha}{\phi(\alpha)} \mathrm{e}^{-\alpha K} \mathbb{E} S^{K, c}\left(T_{0}\right) \\
& -\frac{\alpha}{\phi(\alpha)} \mathrm{e}^{-\alpha K} \mathbb{E}\left[\sum_{0 \leq s \leq T_{0}} \frac{1-\mathrm{e}^{-\alpha \Delta S^{K}(s)}}{\alpha}\right] .
\end{aligned}
$$

The first term on the rhs of (27) is readily inverted using Definition 2.1 giving $W(\cdot)$ times a constant. We observe that the second and third terms correspond to LSTs of functions that have no mass on $[0, K)$. For the second term, note that $\alpha / \phi(\alpha)$ is the LST of $W(\cdot)$ having mass on $[0, \infty)$. The term $\mathrm{e}^{-\alpha K} \alpha / \phi(\alpha)$ thus corresponds to the LST of a function with mass only on $[K, \infty)$, completing the analysis of the second term on the rhs of (27). The third term is a product of two transforms, which corresponds to a convolution. Using Lemma 5.1 and the fact that $\mathrm{e}^{-\alpha K} \alpha / \phi(\alpha)$ has mass only on $[K, \infty)$, it follows that the third term on the rhs of (27) does not contribute on $[0, K)$ as well.

Now, dividing by $\mathbb{E} T_{0}$ and applying Laplace inversion to (27), yields

$$
\mathbb{P}\left(Y^{K} \leq x\right)=\frac{\mathbb{E} I^{K}\left(T_{0}\right)}{\mathbb{E} T_{0}} W(x) .
$$

Using normalization, we directly obtain $\mathbb{E} I^{K}\left(T_{0}\right) / \mathbb{E} T_{0}=1 / W(K)$. This completes the derivation of Theorem 2.2.

\section{References}

1. Asmussen, S.: Applied Probability and Queues, 2nd edn. Springer, New York (2003)

2. Asmussen, S., Kella, O.: On optional stopping of some exponential martingales for Lévy processes with or without reflection. Stoch. Process. Appl. 91, 47-55 (2001)

3. Asmussen, S., Pihlsgård, M.: Loss rates for Lévy processes with two reflecting barriers. Math. Oper. Res. 32, 308-321 (2007)

4. Avram, F., Kyprianou, A.E., Pistorius, M.R.: Exit problems for spectrally negative Lévy processes and applications to (Canadized) Russian options. Ann. Appl. Probab. 14, 215-238 (2004)

5. Bae, J., Kim, S., Lee, E.Y.: Average cost under the $P_{\lambda, \tau}^{M}$ policy in a finite dam with compound Poisson inputs. J. Appl. Probab. 40, 519-526 (2003)

6. Bekker, R., Boxma, O.J.: An M/G/1 queue with adaptable service speed. Stoch. Models 23, 373-396 (2007) 
7. Bekker, R., Boxma, O.J., Kella, O.: Queues with delays in two-state strategies and Lévy input. J. Appl. Probab. 45, 314-332 (2008)

8. Bekker, R., Boxma, O.J., Resing, J.A.C.: Lévy processes with adaptable exponent. Adv. Appl. Probab. 41, 177-205 (2009)

9. Bertoin, J.: Lévy Processes. Cambridge University Press, Cambridge (1996)

10. Bertoin, J.: Exponential decay and ergodicity of completely asymmetric Lévy processes in a finite interval. Ann. Appl. Probab. 7, 156-169 (1997)

11. Bingham, N.H.: Fluctuation theory in continuous time. Adv. Appl. Probab. 7, 705-766 (1975)

12. Brockwell, P.J., Resnick, S.I., Tweedie, R.L.: Storage processes with general release rule and additive inputs. Adv. Appl. Probab. 14, 392-433 (1982)

13. Chydzinski, A.: $M / G-G / 1$ oscillating queueing system. Queueing Syst. 42, 255-268 (2002)

14. Cohen, J.W., Rubinovitch, M.: On level crossings and cycles in dam processes. Math. Oper. Res. 2, 297-310 (1977)

15. Dshalalow, J.H.: Queueing systems with state-dependent parameters. In: Frontiers in Queueing: Models and Applications in Science and Engineering, pp. 61-116 (1997)

16. Faddy, M.J.: Optimal control of finite dams: discrete (2-stage) output procedure. J. Appl. Probab. 11, 111-121 (1974)

17. Hubalek, F., Kyprianou, A.E.: Old and new examples of scale functions for spectrally negative Lévy processes. Preprint (2007). arXiv:0801.0393v2

18. Kella, O.: An exhaustive Lévy storage process with intermittent output. Stoch. Models 14, 979-992 (1998)

19. Kella, O., Whitt, W.: Queues with server vacations and Lévy processes with secondary jump input. Ann. Appl. Probab. 1, 104-117 (1991)

20. Kella, O., Whitt, W.: Useful martingales for stochastic storage processes with Lévy input. J. Appl. Probab. 29, 396-403 (1992)

21. Kella, O., Perry, D., Stadje, W.: A stochastic clearing model with a Brownian and a compound Poisson component. Probab. Eng. Inf. Sci. 17, 1-22 (2003)

22. Kruk, L., Lehoczky, J., Ramanan, K., Shreve, S.: An explicit formula for the Skorokhod map on $[0, a]$. Ann. Probab. 35, 1740-1768 (2007)

23. Kyprianou, A.E.: Introductory Lectures on Fluctuations of Lévy Processes with Applications. Universitext. Springer, Berlin (2006)

24. Kyprianou, A.E., Palmowski, Z.: A martingale review of some fluctuation theory for spectrally negative Lévy processes. In: Séminaire de Probabilités XXXVIII, vol. 1857, pp. 16-29 (2005)

25. Kyprianou, A.E., Rivero, V.: Special, conjugate and complete scale functions for spectrally negative Lévy processes. Electron. J. Probab. 13, 1672-1701 (2008)

26. Lee, E.Y., Ahn, S.K.: $P_{\lambda}^{M}$-policy for a dam with input formed by a compound Poisson process. J. Appl. Probab. 35, 482-488 (1998)

27. Nguyen-Ngoc, L., Yor, M.: Some martingales associated to reflected Lévy processes. In: Séminaire de Probabilités XXXVIII, vol. 1857, pp. 42-69 (2005)

28. Pistorius, M.R.: On doubly reflected completely asymmetric Lévy processes. Stoch. Process. Appl. 107, 131-143 (2003)

29. Prabhu, N.U.: Stochastic Storage Processes. Springer, New York (1980)

30. Roughan, M., Pearce, C.E.M.: Martingale methods for analysing single-server queues. Queueing Syst. 41, 205-239 (2002)

31. Rubinovitch, M., Cohen, J.W.: Level crossings and stationary distributions for general dams. J. Appl. Probab. 17, 218-226 (1980)

32. Sigman, K., Ryan, R.: Continuous-time monotone stochastic recursions and duality. Adv. Appl. Probab. 32, 426-445 (2000)

33. Takagi, H.: Queueing Analysis, vol. 1. North-Holland, Amsterdam (1991)

34. Tijms, H.C., van der Duyn Schouten, F.A.: Inventory control with two switch-over levels for a class of M/G/1 queueing systems with variable arrival and service rate. Stoch. Process. Appl. 6, 213-222 (1978)

35. Yeh, L.: Optimal control of a finite dam: average-cost case. J. Appl. Probab. 22, 480-484 (1985)

36. Zolotarev, V.M.: The moment of first passage of a level and the behavior at infinity of a class of processes with independent increments. Teor. Verojatnost. i Primenen. 9, 724-733 (1964) 